\title{
Gutzwiller-Hubbard lattice-gas model with variable density: Application to normal liquid ${ }^{3} \mathrm{He}$
}

\author{
D. Vollhardt \\ Max-Planck-Institut für Physik und Astrophysik, Werner Heisenberg Institut für Physik, Föhringer Ring 6, \\ D-8000 München 40, Federal Republic of Germany \\ P. Wölfle* \\ Technische Universität München, Physik Department, D-8046 Garching, Federal Republic of Germany \\ P. W. Anderson \\ Princeton University, Jadwin Hall, P.O. Box 708, Princeton, New Jersey 08544
}

(Received 12 November 1986)

\begin{abstract}
The results of the Gutzwiller approach to a Hubbard lattice-gas model with a variable density of particles is used to describe the pressure dependence of thermodynamic properties of the ground state of normal liquid ${ }^{3} \mathrm{He}$. The molar volume of the liquid is given by that of the underlying lattice and the filling factor $n=1-\delta$ of the band, where $\delta$ describes the deviation from half-filling. If the lattice is taken as incompressible, one finds that there exists a critical pressure at which a transition to a localized state occurs. The transition is accompanied by a disappearance of $\delta$, i.e., the transition only takes place at exactly half-filling. As the transition is approached, $\delta$ and the density of doubly occupied sites are found to scale. The pressure dependence of the effective mass, the spin susceptibility and the compressibility is calculated. In a second model, the lattice is assumed to be compressible, shifting the critical pressure to much higher values. The on-site repulsion $U$ is related to the microscopic soft-core potential $f_{0}^{s}(r)$, which allows one to calculate the pressure dependence of the effective mass and the spin susceptibility. The absence of a localization transition for pressures of the order of the melting pressure of ${ }^{3} \mathrm{He}$ leads to a smooth pressure dependence of the calculated quantities which are qualitatively borne out by experiment.
\end{abstract}

\section{INTRODUCTION}

Liquid ${ }^{3} \mathrm{He}$ is a strongly correlated Fermi system ${ }^{1}$ which in several respects may be considered the prototype of a Fermi liquid. ${ }^{2}$ This neutral, isotropic, and clean high-density system consists of spherical, hence structureless, atoms which interact via a strong hard-core (i.e., short-range) repulsion and a weak van der Waals attraction.

Recently, the interest in strongly correlated Fermi systems with a strong short-range repulsion, which had previously focused on the physics of liquid ${ }^{3} \mathrm{He}$ and neutron stars, has further increased owing to the unusual properties of the so-called "heavy-fermion systems." 3-5 These material are characterized by a very high effective mass $m^{*}$, a strongly enhanced spin susceptibility $\chi_{s}$, and a Wilson ration $R \propto \chi_{s} / m^{*}$ which is not much different from that of a noninteracting system. These properties of heavy-fermion systems are generally believed to be due to the almost-localized $f$ electrons of the rare-earth or actinide components in the heavy-fermion compounds, which experience a strong intra-atomic repulsion and which may hybridize with the spd conduction electrons. ${ }^{4}$

Yet another example of a strongly correlated Fermi system with apparently similar features is that of electrons in disordered systems close to the metal-insulator transition. ${ }^{6-8}$

In view of the fact that both normal-liquid and superfluid ${ }^{3} \mathrm{He}$ (Refs. 9-13) have been the subject of inten- sive investigation over the past decade, it is only natural that ${ }^{3} \mathrm{He}$ has been used as a testing ground for concepts dealing with strongly correlated Fermi systems. ${ }^{4,5}$

Microscopically, a general property of the strong shortrange repulsion seems to be a suppression of charge fluctuations and an enhancement of (local) spin fluctuations. The latter degrees of freedom are of low energy such that the spin entropy will appear already at a temperature $T_{0}$ much lower than the (renormalized) degeneracy temperature $T_{F}^{*}$. Phenomenologically, a unifying feature enters via the Fermi-liquid behavior, which characterizes the low-temperature thermodynamic properties for $T<T_{0}{ }^{4,5}$

The overall question then arises of how a strong, shortrange repulsion may lead to a large effective mass, a large Pauli spin susceptibility, a Wilson ratio of order unity, and a large Fermi liquid parameter $F_{0}^{s}$, which in the case of ${ }^{3} \mathrm{He}$ (where $F_{0}^{s} \gg F_{1}^{s}$ ) implies a small compressibility.

As first observed by Anderson and Brinkman, ${ }^{11}$ the Gutzwiller variational approach ${ }^{14,15}$ to the Hubbard model, ${ }^{14,16,17}$ obtained by Brinkman and Rice ${ }^{18,19}$ for the metal-insulator transition in a half-filled band, yields results in qualitative agreement with the properties of normal liquid ${ }^{3} \mathrm{He}$. A detailed discussion of this lattice-gas model and of the concept of an almost localized Fermi liquid has been given by Vollhardt. ${ }^{20}$ The lattice-gas model stresses the importance of the fact that ${ }^{3} \mathrm{He}$ atoms want to stay well separated so as to avoid the mutual and short-ranged interaction between the hard cores. Within such a picture the large effective mass of the ${ }^{3} \mathrm{He}$ quasi- 
particles and the small compressibility of the liquid, i.e., the largeness of $F_{0}^{s}$ and $F_{1}^{s}$, can be understood in a natural way.

In the model the hard-core repulsion is described by an interaction which only acts on the same lattice site and if the particles have opposite spin. The effective Hamiltonian has the form

$$
H=\sum_{i j} \sum_{\sigma} t_{i j} c_{i \sigma}^{\dagger} c_{j \sigma}+U \sum_{i} n_{i \uparrow} n_{i \downarrow} .
$$

Here the first term represents the kinetic energy, where $t_{i j}$ is a (nearest-neighbor) hopping constant for $i \neq j$ and $c_{i \sigma}^{\dagger}, c_{i \sigma}$ are the usual fermion creation and destruction operators for particles with spin $\sigma$ at a site $i$. This term is quantum mechanical in origin. The second term describes an on-site interaction, where $U$ is an effective interaction constant and $n_{i \sigma}=c_{i \sigma}^{\dagger} c_{i \sigma}$ are density operators. This lattice model, generally referred to as the Hubbard model, ${ }^{14,16,17}$ was originally introduced to treat electronic correlations and magnetic ordering in narrow-band electron systems where the Coulomb interaction could be assumed to be strongly screened. Note, that in the case of a liquid like ${ }^{3} \mathrm{He}$, the existence of a lattice (whose actual coordination should not, of course, influence the results for the physical properties of the liquid very strongly) serves for two purposes: (i) it describes the well-known short-range, solidlike correlations of the liquid, and (ii) it provides a finite probability for interactions. In this way the finite size of the ${ }^{3} \mathrm{He}$ atoms is simulated. Furthermore, in the original version of the model with equal numbers of particles and lattice sites, the lattice mimics an attractive interaction not present in (1).

In fact, the hard cores of the ${ }^{3} \mathrm{He}$ atoms do not allow two atoms to be strictly at the same lattice site, in contrast to the case of pointlike electrons. We therefore consider the atoms to be distributed in a regular array of cells which, in the case of a solid, are singly occupied. There will be a finite probability for double occupancy of a given cell. This would correspond to an interstitial in the lattice, which, in turn, is associated with the existence of a vacancy. It is then natural to identify the energy of formation of an interstitial with the on-site repulsion $U$ in the model. In the following we will nonetheless use the expression "doubly occupied site" when we actually mean "doubly occupied cell."

A variational solution for the lattice model (1) was proposed by Gutzwiller. ${ }^{14,15}$ He constructed a variational wave function $|\psi\rangle$,

$$
\begin{aligned}
|\psi\rangle & =\prod_{i}\left[1-(1-g) n_{i \uparrow} n_{i \downarrow}\right]\left|\psi_{0}\right\rangle \\
& =g^{\hat{D}}\left|\psi_{0}\right\rangle,
\end{aligned}
$$

given by that of the noninteracting system (i.e., a Slater determinant) $\left|\psi_{0}\right\rangle$, but with a reduced amplitude. Here, $g$ is a variational parameter and $\widehat{D}=\sum_{i} n_{i \uparrow} n_{i \downarrow}$ is a number operator counting the number $D$ of doubly occupied sites on the lattice. Clearly, $D$ will decrease for increasing interaction strength $U$ in order to reduce the overall interaction energy. For this to occur the hopping probability, i.e., the quantum-mechanical kinetic energy, also has to be reduced. The reduction factor $g^{\hat{D}}$ is supposed to take both of these features into account.

Using the wave function $|\psi\rangle$ the ground-state energy has to be calculated from (1). To this end, Gutzwiller ${ }^{14,15}$ introduced an approximation to evaluate the necessary matrix elements as well as the norm $\langle\psi \mid \psi\rangle$. This approximation is equivalent to the calculation of the classical, statistical weighting of different configurations of particles (neglect of relative phases of configurations). It focuses on configurations of spins with different number of doubly occupied sites. Hence, while the interaction term in (1) may be treated exactly using the variational wave function $|\psi\rangle$, the kinetic energy is approximated by neglecting spatial correlations. For a detailed discussion see Ref. 20. The basic idea behind the choice of the variational wave function (2) and the simple approximation for the ground-state energy is, in fact, very close to that of the Jastrow wave function and the correlated basis function approach by Feenberg. ${ }^{21}$ Most recently, it has been shown that the result of the Gutzwiller approximation may also be obtained within a saddle-point approximation to a suitably chosen functional integral representation of the Hubbard model. ${ }^{22}$

The spin configurations used in the above-mentioned approximation are determined by $L, N_{\uparrow}, N_{\downarrow}$, and $D$, the numbers of lattice sites, up spins, down spins, and doubly occupied sites, respectively $\left(n_{\sigma}=N_{\sigma} / L, d=D / L\right)$. In general, $n_{\uparrow} \neq n_{\downarrow}$ and we introduce

$$
\begin{aligned}
& n=n_{\uparrow}+n_{\downarrow}, \\
& m=n_{\uparrow}-n_{\downarrow},
\end{aligned}
$$

where $m$ measures the magnetization of the system and $n=1-\delta$, such that $\delta$ denotes the deviation from the half-filled-band case.

The ground-state energy per lattice site, of the Hubbard model in the Gutzwiller approximation, $\widetilde{E}_{\mathrm{GH}}=E_{\mathrm{GH}} / L$, of the system, is found to be given by ${ }^{15}$

$$
\widetilde{E}_{\mathrm{GH}}=\sum_{\sigma} q_{\sigma} \bar{\varepsilon}_{\sigma}+U d+\frac{\Delta}{2} n \text {. }
$$

Here,

$$
\bar{\varepsilon}_{\sigma}=\sum_{|\mathbf{k}| \leq k_{F \sigma}} \varepsilon(\mathbf{k})<0
$$

is the average energy of the particles with spin $\sigma$ in the uncorrelated state, with $k_{F \sigma}$ as the respective Fermi momentum and

$$
\varepsilon(\mathbf{k})=L^{-1} \sum_{i \neq j} t_{i j} \exp \left[i \mathbf{k} \cdot\left(\mathbf{R}_{i}-\mathbf{R}_{j}\right)\right]
$$

We have added the third term on the right-hand side (rhs) of (5) to make $\widetilde{E}_{\mathrm{GH}}$ positive. It represents an energy shift of the ground-state energy which is independent of the interaction. Assuming a band of energy states of total width $\Delta$, the shift implies that the energy is measured from the lower band edge rather than from the Fermi energy. In this way one also obtains the correct low-density limit $(n \rightarrow 0)$ of the energy for the noninteracting case. The quantities $q_{\sigma}$ represent the respective discontinuities 
in the single-particle occupation probability at the Fermi surface of the correlated system and are given by ${ }^{15}$

$q_{\sigma}=2 \frac{1-\delta+m-2 d}{1-(\delta-m)^{2}}\left[\sqrt{d+\delta}+\left(d \frac{1-\delta-m-2 d}{1-\delta+m-2 d}\right)^{1 / 2}\right]^{2}$

Note that the Gutzwiller approximation has a very attractive feature: it obeys Luttinger's theorem concerning the conservation of the Fermi-surface volume. ${ }^{23}$ Equation (5) for the ground-state energy still has to be minimized with respect to $d$ to determine the optimal number of doubly occupied sites.

In the case of a half-filled, nonmagnetic band $\left(N_{1}\right.$ $=N_{\downarrow}=L / 2$, i.e., $m=\delta=0$ ), Eq. (5) describes a transition at a finite interaction strength $U=U_{c}$ (where $U_{c}=8\left|\bar{\varepsilon}_{0}\right|$ and $\left.\bar{\varepsilon}_{0}=\bar{\varepsilon}_{\uparrow}+\bar{\varepsilon}_{\downarrow}\right)$ at which the lattice sites become singly occupied $(d=0){ }^{18,19}$ At this point the kinetic energy goes to zero (since $q_{\uparrow}=q_{\downarrow}=0$ ) and hence the system is localized (infinite effective mass). As discussed by Anderson and Brinkman ${ }^{11}$ and Vollhardt, ${ }^{20}$ the results for the effective mass, the spin susceptibility, and the compressibility closely resemble the experimentally measured behavior of these quantities for normal liquid ${ }^{3} \mathrm{He}$ in the ground state. In fact, the results may be understood within the concept of a simplified Landau theory. ${ }^{20}$ The effective mass $m^{*}$ and the Landau parameters $F_{0}^{a}$ and $F_{0}^{s}$ are thus obtained as ${ }^{18-20}$

$$
\begin{aligned}
& \frac{m^{*}}{m}=\frac{1}{1-\bar{U}^{2}}, \\
& F_{0}^{a}=-p\left(1-\frac{1}{(1+\bar{U})^{2}}\right), \\
& F_{0}^{s}=p\left(\frac{1}{(1-\bar{U})^{2}}-1\right),
\end{aligned}
$$

where $\bar{U}=U / U_{c}$ and $p \simeq 1$. For $\bar{U} \rightarrow 1$ the effective mass and $F_{0}^{s}$ diverge, while $F_{0}^{a}$ saturates at a small, negative value $\left(F_{0}^{a} \simeq-\frac{3}{4}\right)$. Hence, in the case of ${ }^{3} \mathrm{He}$, where the effective mass is considerably enhanced over its bare value $\left(2.76<m^{*} / m<5.76\right)$, the dimensionless interaction parameter is always close to unity, i.e., ${ }^{3} \mathrm{He}$ is always close to the localization transition ("almost-localized" Fermi liquid). Eliminating the interaction parameter $\bar{U}$ in favor of the pressure-dependent effective mass, (8), allows one to calculate the pressure dependence of $F_{0}^{a}$ and $F_{0}^{s} \cdot{ }^{20}$ One finds that, for increasing pressure, $F_{0}^{a}$ approaches the pressure-independent value $F_{0}^{a} \simeq-\frac{3}{4}$, while $F_{0}^{s}$ grows strongly. In particular, the pressure independence of $F_{0}^{a}$ implies that the pressure dependence of the spin susceptibility

$$
\chi_{s}=\mu_{0}^{2} N(0) \frac{m^{*} / m}{1+F_{0}^{a}}
$$

[ $N(0)$ is the density of states at the Fermi level for both spin projections of a Fermi gas with bare mass] comes almost entirely from that of the effective mass $m^{*}$. Hence the Wilson ratio

$$
\chi_{s} /\left[\mu_{0}^{2} N(0)\left(m^{*} / m\right)\right]=\left(1+F_{0}^{a}\right)^{-1} \simeq 4
$$

is also essentially pressure independent. This shows that the strong enhancement of the spin susceptibility over its Fermi-gas value is not due to an incipient ferromagnetic transition $\left(F_{0}^{a} \rightarrow-1\right)$ as anticipated by paramagnon theory. ${ }^{24}$ The results for $F_{0}^{a}$ and $F_{0}^{s}$ are borne out by experiment: ${ }^{10,25}$ qualitatively so in the case of $F_{0}^{s}$ (i.e., the compressibility), but even quantitatively for $F_{0}^{a}$ (i.e., the spin susceptibility). It tells us that the simple lattice Hamiltonian (1) already describes essential features of the interaction within liquid ${ }^{3} \mathrm{He}$ correctly. In this sense static properties of ${ }^{3} \mathrm{He}$ may be understood to be largely determined by the tendency of the hard cores of the particles to keep well separated, i.e., by an incipient localization of the particles.

Using a totally different approach, qualitatively identical results have been obtained by Pfitzner and Wölfle. ${ }^{26}$ They showed that the Fermi-liquid interaction and the quasiparticle scattering amplitude for an almost-localized Fermi liquid tend to a universal limit characterized by large values of $F_{0}^{s}$ and $F_{1}^{s}$ and small values of $F_{0}^{a}, F_{1}^{a}$, and the higher $l$ components. Their arguments are based on a diagrammatic approach involving the two-particle vertex function in the particle-hole and particle-particle channels. Similar results are obtained within a refined version of the so-called induced interaction model of $\mathrm{Babu}$ and Brown. ${ }^{27-29}$ Most recently, Baeriswyl et al. ${ }^{30}$ using sum rules on charge and spin conductivities, showed that almost localized Fermi liquids in a real lattice have small values of $F_{1}^{a, s}$.

An extension of the lattice-gas model for ${ }^{3} \mathrm{He}$ to finite temperatures was discussed by Seiler et al. ${ }^{31}$ Within the model three different temperature regimes may be distinguished: (i) for $T \ll T_{F}$ the system is a Fermi liquid, (ii) for $T_{F} \leqslant T \leqslant U$ it shows classical behavior, but is still strongly correlated, while (iii) for $T \gg U$ the system behaves free-particle-like. In the second temperature regime the entropy of the almost-localized fermions is bounded by $R \ln 2 /{ }^{3} \mathrm{He}$ atom, because in this temperature range essentially only singly occupied lattice sites exist. The existence of such a bound had already been assumed much earlier by Goldstein ${ }^{32}$ (see also Ref. 33). Within this framework the crossover from Fermi liquid to classical behavior at $T \sim T_{F}^{*}$ has been described, which otherwise is outside the scope of Fermi-liquid theory ${ }^{2}$ itself. A good qualitative agreement with the measured specific heat, spin susceptibility, and thermal-expansion coefficient is obtained. In particular, it is found that the anomalous behavior of the specific heat, i.e., the sharp kink at about $100 \mathrm{mK}$ and the plateaulike structure above this temperature, ${ }^{25}$ is caused by the bound of $R \ln 2$ on the entropy.

\section{VARIABLE FILLING FACTOR}

The results of the original lattice-gas approach to ${ }^{3} \mathrm{He}$ (Refs. 11 and 20) were based on the assumption of a halffilled band $(\delta=0)$. There remained the question of the validity of this assumption, even though the finding of the incipient localization, i.e., the vicinity of the solid phase, where a half-filled band is formed anyhow, makes this as- 
sumption appear reasonable.

In the following we will study the situation where the number of particles is not necessarily equal to the number of lattice sites, i.e., $n=1-\delta \leq 1$. For this purpose $\delta$, the deviation from half-filling, will be considered as a variable itself. Consequently, we need a new equation to determine this variable. It is provided by an equation for the pressure, to be discussed below. We want to understand the behavior of $\delta$ when the external pressure $P$ and/or the interaction $U$ is increased.

We write

$$
\begin{aligned}
1-\delta=\frac{N}{L} & =\frac{N}{V} \frac{V}{L} \\
& =\frac{v_{s}}{v}
\end{aligned}
$$

where $v=V / N$ is the volume per particle in the liquid and $v_{s}=V / L$ is the volume per lattice site. [In the rest of the paper $v$ and $v_{s}$ will be given as molar volumes in units of $\mathrm{cm}^{3}$; note that $v$ translates into an actual volume per particle, $a^{3}\left(\AA^{3}\right)$, by use of the Avogadro number $N_{A}$ : $v\left(\mathrm{~cm}^{3}\right) \hat{=} 0.6022 a^{3}\left(\AA^{3}\right)$.]

In the lattice model the volume per lattice site, $v_{s}=V / L$, is taken as the specific volume of the solid itself. The solid is then defined by $d=0, \delta=0$, i.e., by single occupancy of every lattice site (half-filling). The liquid is characterized by $\delta>0$, i.e., a finite number of unoccupied sites on the lattice. In this way the larger molar volume of the liquid as compared to the solid is naturally taken into account: for $\delta>0$ one has $v>v_{s}$. The molar volume of the liquid is then given in terms of the volume of the solid and the finite filling factor by

$$
v=\frac{v_{s}}{1-\delta}
$$

Since the volume $v$ is a function of the external pressure the volume of the solid, $v_{s}$, and the filling factor, will also be pressure dependent. At this point the lattice-gas approach may be pursued in two different ways: the lattice itself may be assumed to be incompressible ( $v_{s}=$ const). In this case an external pressure would only act on the particles on the lattice while the lattice spacing stays constant. Then the pressure dependence of $v$ comes entirely from that of the filling factor, i.e., $v_{s}=$ const, $\delta=\delta(P)$. Alternatively, and more realistically, the lattice (i.e., the solid) may be treated as compressible itself. In this case both $v_{s}$ and $\delta$ are functions of the external pressure. We note that experimentally the transition from the liquid to the solid occurs via a first-order transition. At the melting pressure $P_{m}=34.36$ bars, one has $v\left(P_{m}\right) \equiv v_{m}=25.54 \mathrm{~cm}^{3}$ and $v_{s}\left(P_{m}\right) \equiv v_{s, m}=24.23 \mathrm{~cm}^{3},{ }^{34}$ and hence $\delta\left(P_{m}\right) \equiv \delta_{m}$ $=0.0513$, showing that at melting pressure the molar volume of the liquid is about $5 \%$ larger than that of the solid across the transition. So at the transition a volume reduction occurs. This effect (as, in fact, the first-order transition itself) is not described in our theory, which is based on the effective Hamiltonian (1) and which does not contain a mechanism responsible for the transition to the solid.

\section{THE PRESSURE}

Using the volume dependence of the ground state energy (5) introduced via (13), we may now define an associated pressure. For this we bear in mind that (5) does not represent the total ground-state energy of the lattice-gas model, since (1) and (5) neither contain an explicit attractive part necessary to hold the system together, nor any realistic repulsive part. Hence, the total energy per lattice site, $\widetilde{E}_{\text {tot }}$, may be written as

$$
\widetilde{E}_{\mathrm{tot}}=\widetilde{E}_{\mathrm{GH}}+\widetilde{E}_{\mathrm{res}},
$$

where $\widetilde{E}_{\text {res }}$ represents the residual contributions to the ground-state energy per lattice site not included in (1). Taking the definition of the external pressure $P$, $P=-\left(\partial E_{\text {tot }} / \partial V\right)_{N=\text { const }}$, we obtain

$$
P=P_{\mathrm{GH}}+P_{\text {res }}
$$

where $P_{\mathrm{GH}}=-\left(\partial E_{\mathrm{GH}} / \partial V\right)_{N}$ and $P_{\text {res }}=-\left(\partial E_{\text {res }} / \partial V\right)_{N}$ is the pressure due to these residual interactions. In general, $E_{\mathrm{GH}}=\widetilde{E}_{\mathrm{GH}} L$ is a function of $v, v_{s}$, and $\delta$ such that

$$
-P_{\mathrm{GH}}=\widetilde{E}_{\mathrm{GH}}\left(\frac{\partial L}{\partial V}\right)_{N}+L\left(\frac{\partial \widetilde{E}_{\mathrm{GH}}}{\partial V}\right)_{N}
$$

and

$$
\begin{aligned}
\left.\frac{\partial}{\partial V} \widetilde{E}_{\mathrm{GH}}\left(v, v_{s}, \delta\right)\right|_{N}= & \frac{1}{N}\left(\frac{\partial \widetilde{E}_{\mathrm{GH}}}{\partial v}\right)_{v_{s}, \delta}+\left(\frac{\partial \widetilde{E}_{\mathrm{GH}}}{\partial v_{s}}\right)_{v, \delta} \frac{\partial v_{s}}{\partial V} \\
& +\left(\frac{\partial \widetilde{E}_{\mathrm{GH}}}{\partial \delta}\right)_{v, v_{s}} \frac{\partial \delta}{\partial V}
\end{aligned}
$$

Using (13) we find

$$
\begin{aligned}
& \frac{\partial L}{\partial V}=\frac{1}{v_{s}}\left(1-\frac{\kappa_{s}}{\kappa}\right), \\
& \frac{\partial v_{s}}{\partial V}=\frac{1}{L} \frac{\kappa_{s}}{\kappa}, \\
& \frac{\partial \delta}{\partial V}=\frac{1}{L v}\left[1-\frac{\kappa_{s}}{\kappa}\right),
\end{aligned}
$$

where

$$
\begin{aligned}
& \kappa_{s}=-\frac{1}{v_{s}}\left(\frac{\partial v_{s}}{\partial P}\right), \\
& \kappa=-\frac{1}{v}\left(\frac{\partial v}{\partial P}\right)
\end{aligned}
$$

are the compressibilities of the lattice (i.e., solid) and of the liquid, respectively.

Combining (15)-(21) leads to an expression for the external pressure in terms of the volume changes of $\widetilde{E}_{\mathrm{GH}}$ and $P_{\text {res }}$ : 


$$
\begin{aligned}
P= & -\frac{1}{v_{s}}\left[1-\frac{\kappa_{s}}{\kappa}\right]\left[\widetilde{E}_{\mathrm{GH}}+(1-\delta)\left(\frac{\partial \widetilde{E}_{\mathrm{GH}}}{\partial \delta}\right]_{v, v_{s}}\right] \\
& -\frac{1}{1-\delta}\left[\frac{\partial \widetilde{E}_{\mathrm{GH}}}{\partial v}\right]_{v_{s}, \delta}-\frac{\kappa_{s}}{\kappa}\left(\frac{\partial \widetilde{E}_{\mathrm{GH}}}{\partial v_{s}}\right]_{v, \delta}+P_{\mathrm{res}} .
\end{aligned}
$$

To proceed further we have to (i) obtain an explicit expression for $\widetilde{E}_{\mathrm{GH}},(5)$, in terms of $v, v_{s}$, and $\delta$, (ii) find the volume dependence of $P_{\text {res, }}$ and (iii), in the case of a compressible lattice, we have to know its compressibility $\kappa_{s}$. Point (i), concerning $\widetilde{E}_{\mathrm{GH}}$, itself amounts to (a) evaluating $\bar{\varepsilon}_{0 \sigma}$, (6), i.e., the average energy of the correlated system, (b) minimizing $\widetilde{E}_{\mathrm{GH}}$ with respect to $d$ and, last$1 y$, in the case of a compressible lattice, (c), determining the volume dependence of the interaction $U$.

In order to evaluate $\bar{\varepsilon}_{0 \sigma}$ we need the density of states due to the motion of the particles on the lattice. This point has been discussed in detail in Ref. 20. There it was shown that the results of the Gutzwiller approximation are largely independent of the actual lattice type used for the calculation - an important feature for the applicability of a lattice-gas model to describe a liquid. In fact, the lattice enters via a dimensionless parameter $p=2\left|\bar{\varepsilon}_{0}\right| N(0) v_{s}$, where $N(0)$ is the density of states (DOS) at the Fermi surface (in Ref. 20, $v_{s} \equiv 1$ ). This parameter is always close to unity: for a constant DOS, $p=1$; for a Hubbard ellipse, $p=1.08$; and for free fermions, $p=1.2$. For computational reasons, i.e., to enable one to calculate analytically, here we will use a constant DOS. This implies that for the calculation of properties which are determined by the DOS close to the Fermi energy, we assume particle-hole symmetry. We have checked other types of DOS's, finding only (slight) quantitative but not qualitative differences in the final results.

Using a constant DOS of width $\Delta$ the average energy of the uncorrelated system of $\sigma$ spins with general band filling $n=1-\delta$ and magnetization $m$ is found as

$$
\bar{\varepsilon}_{\sigma}=-\frac{1}{2}\left|\bar{\varepsilon}_{0}\right|\left(1-\bar{\mu}_{\sigma}^{2}\right),
$$

where

$$
\begin{gathered}
\bar{\mu}_{\sigma}=-\delta+\sigma m, \\
\left|\bar{\varepsilon}_{0}\right|=\Delta / 4 .
\end{gathered}
$$

In the paramagnetic case

$$
\bar{\varepsilon}=\bar{\varepsilon}_{\uparrow}+\bar{\varepsilon}_{\downarrow}=-\left|\bar{\varepsilon}_{0}\right|\left(1-\delta^{2}\right) .
$$

Concerning the actual values of $\left|\bar{\varepsilon}_{0}\right|$ and $N(0)$, we choose the Fermi-gas values

$$
\begin{aligned}
\left|\bar{\varepsilon}_{0}\right| & =\left|\frac{3}{5} E_{F}-E_{F}\right| \\
& =\frac{2}{5} E_{F}, \\
N(0) & =\frac{3}{2} \frac{E_{F}}{v_{s}}
\end{aligned}
$$

to be in accord with the usual Fermi-liquid theory; at melting pressure $\left|\bar{\varepsilon}_{0}\right| \simeq 2.1 \mathrm{~K}$. Note that $E_{F}$ appearing in (27) and (28) is the Fermi energy for the system at $\delta=0$, i.e., for the solid volume: $E_{F}=\left(3 \pi^{2} / v_{s}\right)^{2 / 3} / 2 m$.
$\widetilde{E}_{\mathrm{GH}}$ is then given by

$$
\widetilde{E}_{\mathrm{GH}}=-\frac{1}{2}\left|\bar{\varepsilon}_{0}\right| \sum_{\sigma} q_{\sigma}\left[1-(\delta-\sigma m)^{2}\right]+U d+2\left|\bar{\varepsilon}_{0}\right| n .
$$

In the nonmagnetic case $\left(m=0, q_{\sigma} \equiv q\right)$ we have

$$
\widetilde{E}_{\mathrm{GH}}=-q\left|\bar{\varepsilon}_{0}\right|\left(1-\delta^{2}\right)+U d+2\left|\bar{\varepsilon}_{0}\right|(1-\delta),
$$

where $q^{-1}$ is the effective mass. In this situation it is very convenient to introduce a new variable $x$,

$$
x=\sqrt{d+\delta}+\sqrt{d},
$$

which may be used to replace $d$ in (30). So $\widetilde{E}_{\mathrm{GH}}(d, \delta)$ now becomes a function of $x^{2}, \delta$ with

$$
d=\left(\frac{x^{2}-\delta}{2 x}\right)^{2}
$$

while the expression for $q$ is greatly simplified:

$$
q=\left(\frac{m^{*}}{m}\right)^{-1}=1-\frac{\left(1-x^{2}\right)^{2}}{1-\delta^{2}} .
$$

Equation (16) then reduces to

$\widetilde{E}_{\mathrm{GH}}=\left|\bar{\varepsilon}_{0}\right|\left[(1-\delta)^{2}+\left(1-x^{2}\right)^{2}\right]+U\left(\frac{x^{2}-\delta}{2 x}\right)^{2}$.

Equation (29), in conjunction with (31), may be used to calculate the spin susceptibility $\chi_{s}$,

$$
\chi_{s}^{-1}=\left.\frac{1}{\mu_{0}^{2}} \frac{\partial^{2} \widetilde{E}_{\mathrm{GH}}}{\partial m^{2}}\right|_{m=0},
$$

which is found as

$$
\chi_{s}=\frac{1}{2\left|\bar{\varepsilon}_{0}\right| v_{s}} \frac{2 x^{2}-x^{4}-\delta^{2}}{x^{4}-\delta^{2}} .
$$

Comparing this result with the expression (11) from Fermi-liquid theory yields an expression for the Landau parameter $F_{0}^{a}$,

$$
F_{0}^{a}=-1+p\left(\frac{m^{*}}{m}\right)^{2} \frac{x^{4}-\delta^{2}}{1-x^{2}},
$$

where we take $p=2 N(0)\left|\bar{\varepsilon}_{0}\right| v_{s}=1.2$ as discussed below Eq. (26).

Returning to the nonmagnetic case (34), we may now rewrite the equation for the pressure, (22). The first term on the rhs of (22), expressing the $\delta$ dependence, reads

$$
-\left[\widetilde{E}_{\mathrm{GH}}+(1-\delta) \frac{\partial \widetilde{E}_{\mathrm{GH}}}{\partial \delta}\right] \equiv 8\left|\bar{\varepsilon}_{0}\right| \bar{P},
$$

with

$$
\bar{P}=\frac{1}{8}\left[x^{2}\left[x^{2}+\frac{4\left(1-x^{2}\right)}{x^{2}+\delta}\right]-\delta(2-\delta)\right] .
$$

On the other hand, the $v$ dependence of $\widetilde{E}_{\mathrm{GH}}$ only enters via a $v$ dependence of $U$ : 


$$
\frac{\partial \widetilde{E}_{\mathrm{GH}}}{\partial v}=\frac{\partial U}{\partial v} d,
$$

while the $v_{s}$ dependence is due to $\left|\bar{\varepsilon}_{0}\right|$ and $U$ :

$$
\frac{\partial \widetilde{E}_{\mathrm{GH}}}{\partial v_{s}}=-\frac{8\left|\bar{\varepsilon}_{0}\right|}{v_{s}}\left(\bar{P}^{\prime}-\frac{v_{s}}{8\left|\bar{\varepsilon}_{0}\right|} \frac{\partial U}{\partial v_{s}} d\right),
$$

with

$$
\bar{P}^{\prime}=\frac{1}{12}\left[(1-\delta)^{2}+\left(1-x^{2}\right)^{2}\right] .
$$

So we find, from (22),

$$
\begin{aligned}
P= & \frac{8\left|\bar{\varepsilon}_{0}\right|}{v_{s}}\left[\left(1-\frac{\kappa_{s}}{\kappa}\right] \bar{P}+\frac{\kappa_{s}}{\kappa} \bar{P}^{\prime}\right] \\
& -\left[\frac{\kappa_{s}}{\kappa} \frac{\partial U}{\partial v_{s}}+\frac{1}{1-\delta} \frac{\partial U}{\partial v}\right]\left(\frac{x^{2}-\delta}{2 x}\right)^{2}+P_{\text {res }} .
\end{aligned}
$$

\section{MINIMIZATION OF THE GROUND-STATE ENERGY}

The minimization of (30) with respect to $d$ may now easily be performed by using (34) and minimizing with respect to $x$; one finds

$$
\bar{U}=\frac{x^{4}\left(1-x^{2}\right)}{x^{4}-\delta^{2}}
$$

with $\bar{U}=U /\left(8\left|\bar{\varepsilon}_{0}\right|\right)$. It is an implicit equation for $d$ as a function of the interaction $U$ and the band filling. Introducing

$$
\xi=\delta / x^{2},
$$

with $0 \leq \zeta \leq 1$ and $\zeta=0$ for $\delta=0, d \neq 0$ and $\zeta=1$ for $d=0, \delta \neq 0,(44)$ reduces to the useful result

$$
\bar{U}=\frac{1-x^{2}}{1-\zeta^{2}}
$$

which allows for a discussion of various limits:

(i) For $\delta=0$ and $d \neq 0$ one obtains $d=\frac{1}{4}(1-\bar{U})$, the result for the half-filled band case, i.e., localization occurs at a finite interaction strength $U=U_{c}$.

(ii) For $d=0$ and $\delta \neq 0$, the only solution is $\bar{U}=\infty$, i.e., localization is only possible for infinitely strong repulsion.

(iii) For $d<<\delta$ one finds

$$
d=\frac{\delta(1-\delta)^{2}}{4 \bar{U}^{2}} .
$$

(iv) Lastly, (46) allows for a solution where both $d$ and $\delta$ go to zero. A solution with $\delta \propto d^{\lambda}, \lambda>1$, such that $\zeta=0$ at the transition, is only allowed in the special case $\bar{U}=1$, where $\lambda=3$. However, for general values of $\bar{U}>1$, (46) leads to a solution where $\lambda=1$, such that $\delta / d$ has a constant ratio and $\xi$ assumes a nonzero value

$$
\zeta_{c}=\lim _{d, \delta \rightarrow 0} \zeta=\sqrt{1-1 / \bar{U}}
$$

and hence

$$
d=\frac{\left(1-\zeta_{c}\right)^{2}}{4 \zeta_{c}} \delta
$$

In this case $d$ and $\delta$ scale with each other as the transition is approached. At this point the effective mass also diverges,

$$
\frac{m^{*}}{m} \simeq \frac{\zeta_{c}}{2 \delta} .
$$

Furthermore, close to the transition the Landau parameter $F_{0}^{a},(37)$, goes to a constant value:

$$
\left.F_{0}^{a}\right|_{c}=-\frac{3}{4}-\left(\frac{\zeta_{c}}{2}\right)^{2} \text {. }
$$

For $\zeta_{c} \ll 1$, implying $\delta \ll d \ll 1$ and $\bar{U} \gtrsim 1$, one obtains $\left.F_{0}^{a}\right|_{c} \simeq-\frac{3}{4}$, while for $\zeta_{c} \leqslant 1$, implying $d<<\delta<<1$ and $\bar{U} \gg 1$, one has $\left.F_{0}^{a}\right|_{c} \gtrsim-1$, in which case the system would tend to be ferromagnetic (Nagoaka effect). Note that $-1 \leq F_{0}^{a} \leq 0$ for a repulsive interaction.

\section{INCOMPRESSIBLE LATTICE}

We first study the case of an incompressible lattice, where an external pressure only acts on the particles but not on the lattice itself. In this situation $v_{s}=$ const and we choose $v_{s}$ to be given by that of the solid molar volume at melting pressure: $v_{s, m}=v_{s}\left(P_{m}\right)=24.23 \mathrm{~cm}^{3}$. Consequently, the compressibility $\kappa_{s}=0,\left|\bar{\varepsilon}_{0}\right|$ is constant, and the interaction $U$ may also be taken as constant. The equation for the pressure, (43), then reduces to

$$
P=\frac{8\left|\bar{\varepsilon}_{0}\right|}{v_{s, m}} \bar{P}+P_{\mathrm{res}},
$$

where $8\left|\bar{\varepsilon}_{0}\right| / v_{s, m}=72.01$ bars. To be able to make use of (52), we have to know $P_{\text {res }}$. As discussed earlier, $P_{\text {res }}$ is supposed to describe the pressures due to interactions not contained in the on-site repulsion. At low enough external pressures, i.e., large molar volumes, this interaction will have to be mainly attractive to keep the system together. Therefore $P_{\text {res }}$ will be negative for large $v$. On the other hand, for large external pressures, i.e., small molar volumes, other repulsive interactions than the on-site repulsion may enter. Therefore $P_{\text {res }}$ is expected to increase with decreasing molar volume and may even become positive, acting against the external pressure. Since we have not yet found a model type of interaction in the lattice-gas model which would give a good description of this behavior, we will have to parametrize $P_{\text {res }}$. For this a virial type of expansion in terms of $v^{-n}, n \geq 2$, appears to be most natural. So we make the simple ansatz

$$
P_{\mathrm{res}}=\frac{\alpha}{v^{2}}+\frac{\beta}{v^{3}}
$$

which clearly allows for the features of $P_{\text {res }}$ expected to occur. Hence, the pressure in (52) is determined by the three quantities $x^{2}, \delta$, and $v$. Together with the equation for $\bar{U},(44)$, and the one connecting $v$ and $\delta$, 


$$
v=\frac{v_{s, m}}{1-\delta},
$$

this gives three equations for four unknowns, allowing us to establish a unique $P(v)$ relation. The three unknown constants $\alpha, \beta$, and $\bar{U}$ in (53) and (44), respectively, may be determined by fitting to three parameters. For this we choose the molar volumes at $P=0$ and $P_{m}$ and the effective mass at $P_{m}$. So, including $v_{s, m}$ in (54), there are, in total, four input parameters:

$$
\begin{aligned}
& \text { (i) } v_{s, m}=v_{s}\left(P_{m}\right)=24.23 \mathrm{~cm}^{3}, \\
& \text { (ii) } v_{0}=v(P=0)=36.84 \mathrm{~cm}^{3}, \\
& \text { (iii) } v_{m}=v\left(P_{m}\right)=25.54 \mathrm{~cm}^{3}, \\
& \text { (iv) } \frac{m^{*}}{m}\left(P_{m}\right)=5.76 .
\end{aligned}
$$

From these values we find $\alpha=-5.62 \times 10^{4}$ bars $\mathrm{cm}^{6}$, $\beta=1.67 \times 10^{6}$ bars $\mathrm{cm}^{9}$, while $\bar{U}=1.3157$, corresponding to an interaction parameter $U=21 \mathrm{~K}$. Note that while at melting pressure $\delta_{m}=0.0513$, at $P=0$ one has $\delta_{0} \equiv \delta(P=0)=0.3423$, i.e., the $30 \%$ volume increase of the liquid from melting pressure to saturated vapor pressure is fully contained in $\delta$ because the solid is assumed incompressible.

We are now able to calculate the full pressure dependence of $\delta$, the density of doubly occupied sites $d$, the effective mass $m^{*}$, the spin susceptibility $\chi_{s}$, the Landau parameter $F_{0}^{a}$, the molar volume $v$, and the compressibility $\kappa$, which in Fermi-liquid theory is related to the Landau parameter $F_{0}^{s}$ by

$$
\begin{aligned}
\kappa & =v^{2} \frac{\partial n}{\partial \mu} \\
& =\kappa_{N} \frac{m^{*} / m}{1+F_{0}^{s}},
\end{aligned}
$$

where $\mu$ is the chemical potential and

$$
\kappa_{N}=v^{2} N(0)
$$

is the compressibility of the Fermi gas.

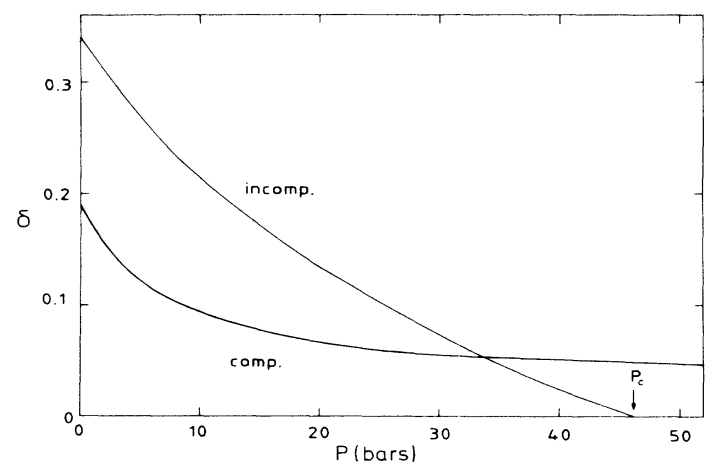

FIG. 1. $\delta$, the deviation from half-filling $(n=1-\delta)$, is shown as a function of pressure $P$. The labels "incomp." and "comp." indicate the results for the incompressible and compressible lattice, respectively.

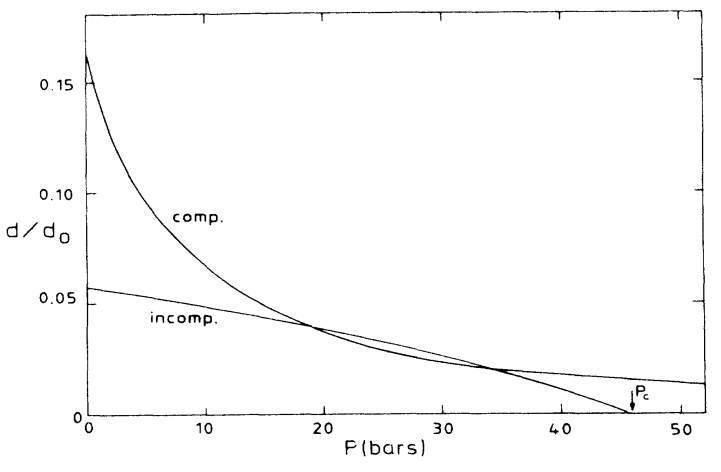

FIG. 2. $d$, the density of doubly occupied sites $(d=D / L)$, normalized to $d_{0}=\left(\frac{1}{2} n\right)^{2}$, the corresponding value of $d$ for the noninteracting case, is shown as a function of pressure. (incomp., incompressible lattice; comp., compressible lattice).

The result for $\delta$ as a function or pressure for the incompressible case is shown in Fig. 1 (curve labeled "incomp."; this label is also used in the other figures to distinguish the results for the incompressible lattice from those for the compressible lattice to be discussed later). The deviation from half-filling decreases monotonically from $\delta_{0}=0.3423$ to $\delta_{m}=0.0513$ at melting pressure and becomes zero at a hypothetical, critical pressure $P_{c} \simeq 46$ bars.

In Fig. 2 the corresponding curve for $d$, the density of doubly occupied sites, normalized to $d_{0}=\left(\frac{1}{2} n\right)^{2}$, the value of $d$ in the noninteracting system, is shown. It also decreases monotonically from $d / d_{0}=0.058$ at $P=0$ to $d / d_{0}=0.020$ at melting pressure. At the critical pressure where $\delta$ vanishes, $d$ also becomes zero, i.e., the system localizes. Hence, the transition to a state with $\delta=0$ occurs simultaneously with the localization transition of the particles $(d=0)$. This behavior becomes evident from Fig. 3, where $d$ vs $\delta$ is plotted. We see that, as $P_{c}$ is approached, both $d$ and $\delta$ vanish at the same rate, i.e., the two parameters scale,

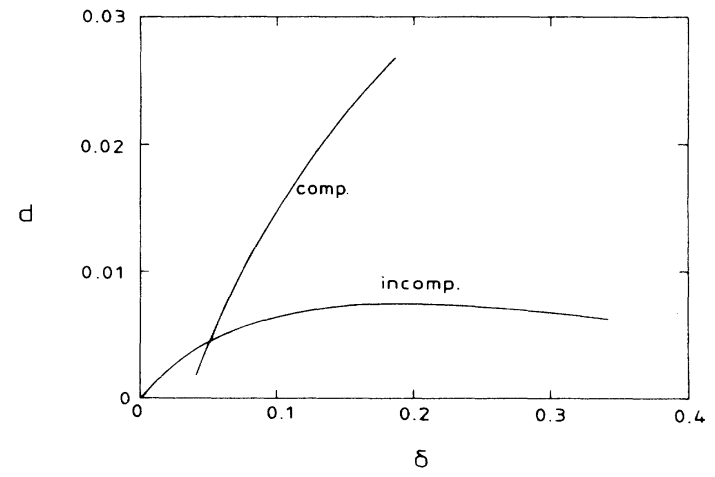

FIG. 3. $d$ vs $\delta$ for the two models (incomp., incompressible lattice; comp., compressible lattice). 


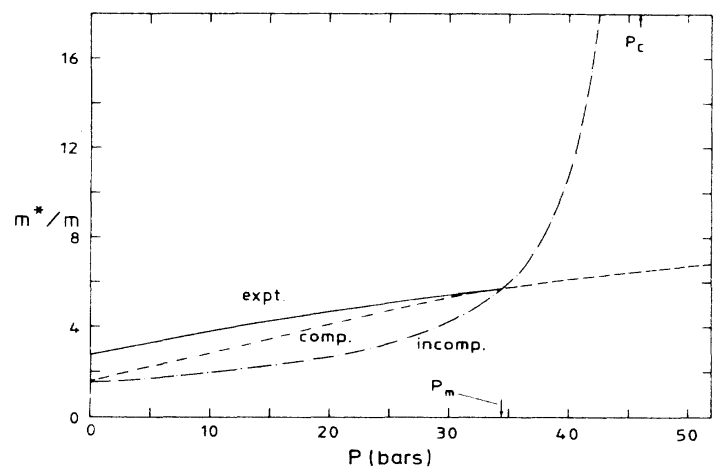

FIG. 4. The effective mass $m^{*} / m$ of liquid ${ }^{3} \mathrm{He}$, vs pressure [expt., experimental curve (Ref. 25); incompr., theoretical result for an incompressible lattice; comp., compressible lattice]. The pressure $P_{m}$ indicates the melting pressure of liquid ${ }^{3} \mathrm{He}$, while $P_{c}$ is the critical pressure where the transition $d, \delta \rightarrow 0$ occurs in the case of an incompressible lattice.

$$
\lim _{P \rightarrow P_{c}} \frac{\delta}{d}=\left(\frac{2 \zeta_{c}}{1-\zeta_{c}}\right)^{2}=3.69,
$$

where $\zeta_{c}=[(\bar{U}-1) / \bar{U}]^{1 / 2}=0.490$. So, as $P_{c}$ is approached, $\delta \propto d$, such that at the transition the band will actually be half-filled. We mention in passing that the scaling of $d$ and $\delta$ implies a smooth, analytic $\delta$ dependence of the ground-state energy even in the limit $d, \delta \rightarrow 0$.

The pressure dependence of the effective mass $m^{*}$ is shown in Fig. 4. At $P=0, m^{*} / m=1.52$, i.e., the enhancement is smaller than that measured experimentally (where $m^{*} / m=2.76$ ). As the pressure increases, $m^{*}$ increases also with about the same slope as the experimental $m^{*}$. Close to the melting pressure, the increase of $m^{*}$ becomes larger and, at $P_{c}$ where $d$ and $\delta$ vanish, $m^{*}$ diverges at a rate given by (50).

The $v(P)$ curve is shown in Fig. 5. The pressure dependence of $v$ is well reproduced, in spite of the simplicity of virial-type approximation for $P_{\text {res }}$ in (52). The compressibility $\kappa,(21 \mathrm{~b})$, is obtained from (52) as

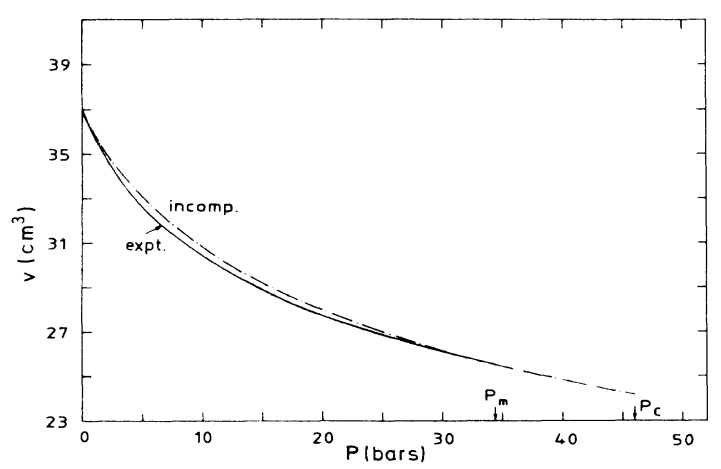

FIG. 5. The molar volume of normal liquid ${ }^{3} \mathrm{He}$ in the limit $T \rightarrow 0$ as a function of pressure. [expt., experimental curve (Ref. 25 ); incompr., theoretical result for an incompressible lattice].

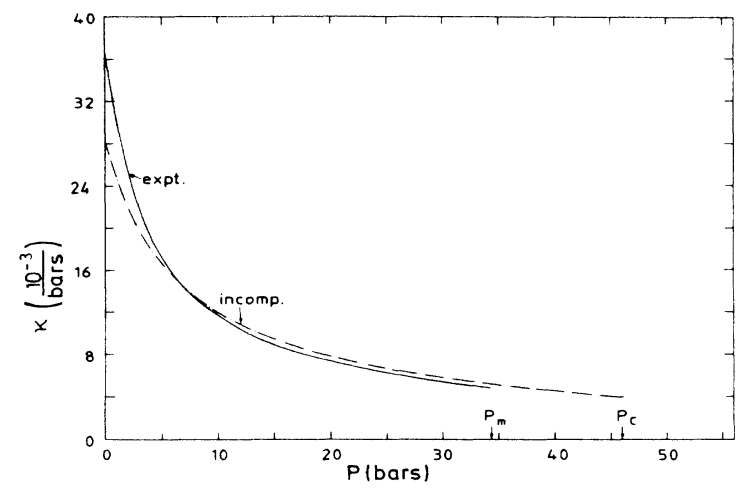

FIG. 6. The compressibility $\kappa$ of liquid ${ }^{3} \mathrm{He}$, vs pressure [expt., experimental curve (Ref. 25); incomp., theoretical result for an incompressible lattice].

$$
\kappa=\frac{v_{s}}{8\left|\bar{\varepsilon}_{0}\right|}\left[\frac{2 \alpha}{v^{2}}+\frac{3 \beta}{v^{3}}-(1-\delta) \frac{\partial \bar{P}}{\partial \delta}\right)^{-1},
$$

where

$$
\begin{aligned}
\frac{\partial \bar{P}}{\partial \delta}= & -\frac{1}{4}(1-\delta)-\frac{1}{2} \frac{x^{2}\left(1-x^{2}\right)}{\left(x^{2}+\delta\right)^{2}} \\
& +\frac{\bar{U}}{2} \frac{\delta}{x^{2}}\left[1-\frac{1}{2} x^{2}-\frac{\delta(1+\delta)}{\left(x^{2}+\delta\right)^{2}}\right) /\left(1-\bar{U}-\frac{3}{2} x^{2}\right) .
\end{aligned}
$$

Note, that $\partial \bar{P} / \partial \delta$ approaches a finite value for $P \rightarrow P_{c}$ because of the scaling of $d$ and $\delta$. Therefore $\kappa$ goes to a finite value at the transition to the solid. This is in contrast to the case where a half-filled band was chosen independent of pressure and where $\kappa$ was found to vanish at the transition. ${ }^{11,18-20}$ The resulting curve for $\kappa(P)$ is shown in Fig. 6. The results for $\kappa$ and $m^{*} / m$ imply that, at the transition, the Landau parameter $F_{0}^{s}$ (Fig. 7), determined via (56), also diverges; but now

$$
F_{0}^{s} \propto m^{*} / m,
$$

i.e., $F_{0}^{s}$ diverges less strongly than in the case $\delta=0, d \neq 0$, where $F_{0}^{s} \propto\left(m^{*} / m\right)^{2}$ for $d \rightarrow 0$.

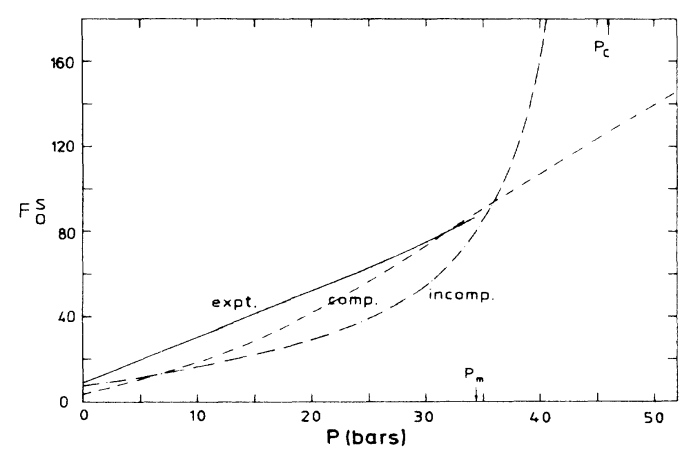

FIG. 7. The Landau parameter $F_{0}^{s}$, entering the compressibility, vs pressure; same abbreviations as in Fig. 4. 


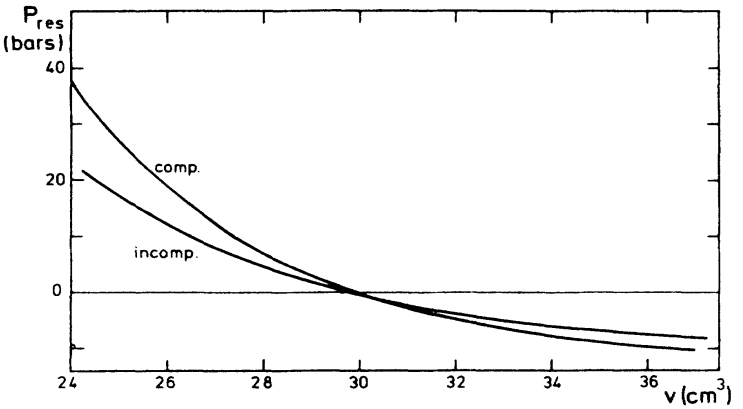

FIG. 8. $P_{\text {res }}$, the pressure due to interactions not described by the Hubbard-type on-site interaction, as a function of molar volume (incomp., incompressible lattice; comp., compressible lattice).

For completeness we show the volume dependence of $P_{\text {res }}$ (Fig. 8), the pressure due to interactions other than the on-site interaction. As anticipated, $P_{\text {res }}<0$ for large volume, indicating the attractiveness of this interaction, while for small volumes $P_{\text {res }}>0$, which characterizes additional repulsion in the system directed against the external pressure.

Finally, in Fig. 9 the pressure dependence of the spin susceptibility is shown. It increases strongly with increasing pressure (with a curvature opposite to that measured experimentally) and diverges at $P_{c}$. However, the divergence is only due to that of the effective mass. This can be seen from the values of the Landau parameter $F_{0}^{a},(11)$, obtained from (37) (see Fig. 10). One indeed finds an essentially pressure-independent $F_{0}^{a}$ which starts from $F_{0}^{a}=-0.73$ at $P=0$, goes to -0.77 at melting pressure, and then approaches its limiting value given by (51), $\left.F_{0}^{a}\right|_{c}=-0.81$.

Hence, the results obtained from the lattice-gas model discussed above, in which particles interact on an incompressible lattice, do reproduce all essential features of the pressure-dependent effective mass, compressibility, and spin susceptibility of normal liquid ${ }^{3} \mathrm{He}$ in the limit

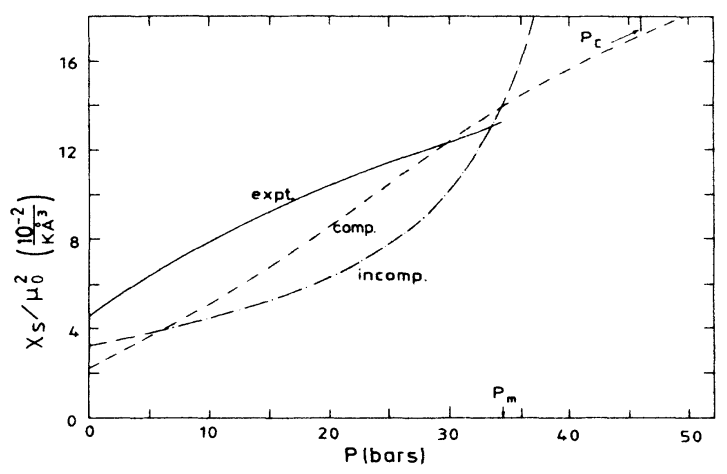

FIG. 9. The spin susceptibility $\chi_{s}$, in units of $\mu_{0}^{2}$, as a function of pressure [expt., experimental curve (Ref. 25); incomp., theoretical result for the incompressible lattice; comp., compressible lattice].

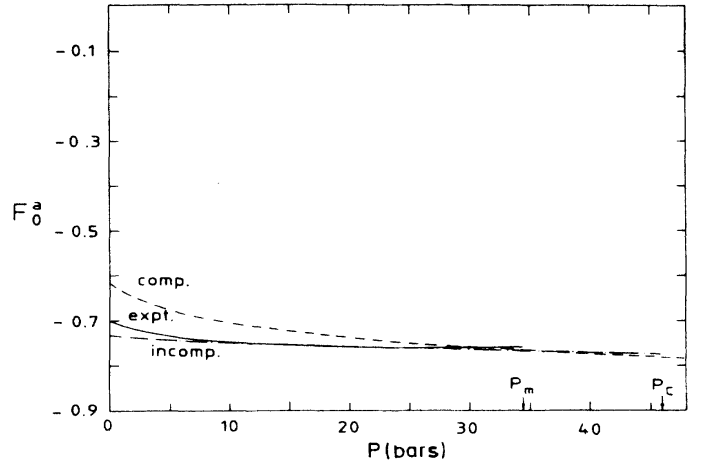

FIG. 10. The Landau parameter $F_{0}^{a}$, entering the spin susceptibility, as a function of pressure; same abbreviations as in Fig. 9.

$T \rightarrow 0$. In particular, we found that at the localization transition, where $d=0$, the system also goes to half-filling $(\delta=0)$, such that $\delta / d=$ const.

\section{COMPRESSIBLE LATTICE}

So far we have assumed that an external pressure only acts on the particles on the lattice but leaves the lattice itself unchanged. This is somewhat unrealistic since even a solid (i.e., the lattice with $d, \delta=0$ ) has a finite compressibility $\kappa_{s}$. If we want to include this effect, two questions arise: (i) What is the compressibility of the lattice for $0 \leq P \leq P_{m}$, and (ii) how does the on-site interaction $U$ change with pressure? To account for the lattice compressibility we take the pressure dependence of the molar volume of solid ${ }^{3} \mathrm{He},{ }^{35,36} v_{s}(P)$, as presented by Hetherington et al. ${ }^{37}$ (solid line $v_{s}$ in Fig. 11), and extrapolate it smoothly to $\boldsymbol{P}=0$ [indicated by the dashed curve $\left(v_{s}\right)$ in Fig. 11]. Note, that in the incompressible case $v_{s}$ was approximated by the horizontal line $v_{s}=$ const. The extrapolation itself is quite arbitrary, but it will suffice to describe the new features introduced by a finite compressibility of the lattice. The pressure dependence of $v_{s}$ for $P \lesssim 100$ bars is well approximated by the expression

$$
v_{s}=v_{s, m}\left[\frac{P-C}{A}\right)^{B} \text {, }
$$

with $A=60.44$ bars, $B=-0.254$, and $C=-26.082$ bars.

Once we allow for a compressible lattice, i.e., for a variable lattice spacing as a function of pressure, the interaction $U$ between the particles also has to be considered pressure dependent. One can get an idea of the pressure dependence of the effective interaction $U$ by considering the following picture: Let us form a doubly occupied cell by displacing an atom from a singly occupied cell. The energy required for this process may be approximated by the difference in the chemical potential of the actual system, $\mu$, and that of a fictitious system without any vacancies, $\mu+\delta \mu$, where an additional particle necessarily leads to the formation of a doubly occupied cell. The difference, $\delta \mu$, is then given by $\delta \mu=v_{c}^{-1}(\partial \mu / \partial n)$, where 


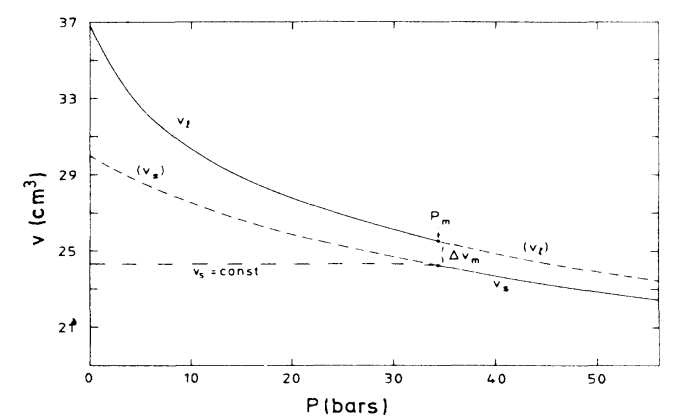

FIG. 11. The molar volumes of liquid, $v_{l}$, and solid, $v_{s},{ }^{3} \mathrm{He}$. Both the curve for the liquid, $\left(v_{l}\right)$ for $P>P_{m}$, and that for the solid, $\left(v_{s}\right)$ for $P<P_{m}$, are smooth extrapolations of the respective experimentally measured curve (see text). At the melting pressure $P_{m}$ the molar volumes of the solid and the liquid differ by $\Delta v \simeq 1.3 \mathrm{~cm}^{3}$.

$v_{c}$ is a volume characterizing the difference in density of the two systems. In addition, we have to take into account the energy of attraction, $|W|$, in the system which one has to work against. Hence,

$$
U=|W|+\frac{1}{v_{c}} \frac{\partial \mu}{\partial n} .
$$

We note that $\partial \mu / \partial n$ is directly related to the compressibility $\kappa$ via (55),

$$
\frac{\partial \mu}{\partial n}=\frac{1}{N(0)} \frac{1+F_{0}^{s}}{m^{*} / m} .
$$

Using the quasiparticle interaction ${ }^{2} f_{0}^{s}(q=0)=F_{0}^{s} /$ $N^{*}(0)$, where $N^{*}(0)=N(0)\left(m^{*} / m\right)$, we find

$$
U=|W|+\frac{1}{v_{c}} f_{0}^{s}(q=0) .
$$

This expression can also be derived from a more microscopic point of view. Namely, we may relate the shortrange (effectively zero-range) interaction $U$ to the soft-core part of the polarization potential $f_{0}^{s}(\boldsymbol{r})$, introduced by $\mathrm{Al}-$ drich and Pines. ${ }^{38}$

The radial dependence of $f_{0}^{s}(r)$ is shown in Fig. 12 . For $r \leq r_{c}$ the potential is given by an effective soft-core repulsion which is supposed to represent the reduction of the bare hard-core repulsion caused by the short-range correlations. For $r>r_{c}$, i.e., the long-range part, the potential is attractive and is assumed as unchanged by the short-range correlations. This behavior may be parametrized by ${ }^{38}$

$f_{0}^{s}(r)=\left\{\begin{array}{l}A\left[1-\left(r / r_{c}\right)^{8}\right], \quad r \leq r_{c} \\ K(14.5,6) \varepsilon\left[\left(\frac{r_{0}}{r}\right)^{14.5}-\left(\frac{r_{0}}{r}\right)^{6}\right], \quad r>r_{c}\end{array}\right.$

where

$K(m, 6)=\left[\left(\frac{6}{m}\right]^{m /(m-6)}-\left[\frac{6}{m}\right]^{6 /(m-6)}\right]^{-1}$

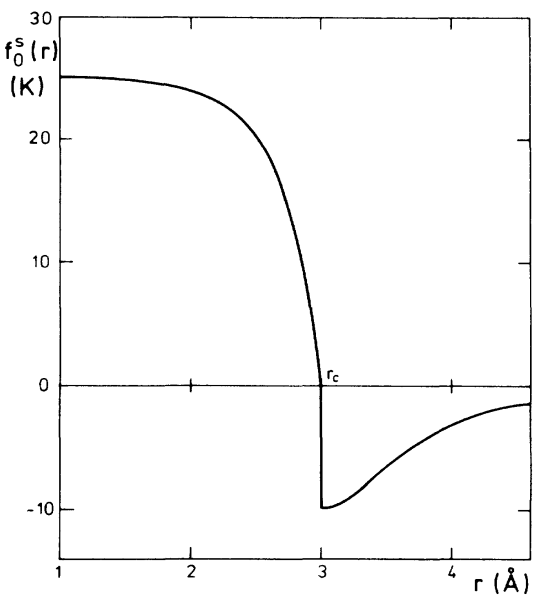

FIG. 12. The radial dependence of the soft-core potential $f_{0}^{s}(r)$ (Ref. 38).

has been introduced by Bennewitz et al. ${ }^{39}$; here, $K(14.5,6)=-3.18, \varepsilon=-10.65 \mathrm{~K}$, and $r_{0}=2.685 \AA$. [In fact, the expression for the attractive part in (66) was originally only chosen for $r_{c}<r \leq r_{a}, r_{a}=6.3 \AA$, while for $r>r_{a}$ the shape of the potential is somewhat different. ${ }^{38,39}$ However, these details are unimportant here.]

Now,

$$
f_{0}^{s}(q=0)=4 \pi \int_{0}^{\infty} d r r^{2} f_{0}^{s}(r) .
$$

The integral may be divided into one for $r \leq r_{c}$, which extends over the repulsive part of $f_{0}^{s}(r)$, introducing the parameter $U$,

$$
U=\frac{4 \pi}{v_{c}} \int_{0}^{r_{c}} d r r^{2} f_{0}^{2}(r),
$$

and one over $r \geq r_{c}$, i.e., the attractive part of $f_{0}^{s}(r)$ :

$$
W=\frac{4 \pi}{v_{c}} \int_{r_{c}}^{\infty} d r r^{2} f_{0}^{s}(r) .
$$

Here, $v_{c}=\frac{4}{3} \pi r_{c}^{3}$. Hence, (68) yields

$$
f_{0}^{s}(q=0)=v_{c}(U-|W|),
$$

or

$$
U=|W|+\frac{1}{v_{c}} \frac{v^{2}}{\kappa} .
$$

This equation is identical to (65), obtained from simple arguments about the energy necessary to insert a particle into a bath of others. The pressure dependence of $U$ is then directly given in terms of the compressibility $\kappa$ of the liquid and

$$
\frac{\partial U}{\partial v}=\frac{2}{\kappa} \frac{v}{v_{c}}\left(1-\frac{1}{2} \frac{d \ln \kappa}{d \ln v}\right) .
$$

Note that the volume dependence of $W$ may be neglected because $W$ is approximately given by the long-range part of the bare potential. The $P-v$ equation for the compressible case, (43), is then obtained as 


$$
\begin{aligned}
P= & \frac{8\left|\bar{\varepsilon}_{0}\right|}{v_{s}}\left[\bar{P}-\frac{\kappa_{s}}{\kappa}\left(\bar{P}-\bar{P}^{\prime}\right)\right] \\
& -\frac{2 v}{\kappa v_{c}(1-\delta)}\left[1-\frac{1}{2} \frac{d \ln \kappa}{d \ln v}\right)\left(\frac{x^{2}-\delta}{2 x}\right)^{2}+P_{\text {res }}(v),
\end{aligned}
$$

where $8\left|\bar{\varepsilon}_{0}\right| / v_{s}=1.461 \times 10^{4} v_{s}^{-5 / 3}$ bars and $\kappa_{s}$ are determined by the assumed pressure dependence of the lattice, (62). In contrast to the case of the incompressible lattice, the pressure equation (74) contains $\kappa$ and $d \kappa / d v$ and is thus a second-order differential equation for $\boldsymbol{P}(v)$. In principle, one could now again make a virial-type ansatz for $\boldsymbol{P}_{\text {res }}$ in terms of $1 / v$ with unknown coefficients which would have to be fitted using some experimental input. On the other hand, there are now at least two more unknown parameters in the equation for $P\left[r_{c}\right.$ and an integration constant, e.g., $\kappa(P=0)$ ], than in the incompressible case. Therefore we do not attempt to solve the differential equation (74). Rather we take the experimentally known $P(v)$ curve $^{25}$ as input, which allows us to determine $U$ via (72). Together with (44), relating $U$ to $d$ and $\delta$, and (13), relating $v$ and $\delta$, we may then calculate the full presence dependence of $\mathrm{m}^{*} / \mathrm{m}$, the susceptibility and the related Landau parameters.

If we take the experimental compressibility at melting pressure, ${ }^{25} \kappa_{m}=4.76 \times 10^{-3}$ bar $^{-1}$, and $r_{c}=3 \AA,{ }^{38}$ we find a value of $U$, which in units of $8\left|\bar{\varepsilon}_{0}\right|$ is given by $\bar{U}\left(P_{m}\right)=1.88$. This is already quite close to $\bar{U}=1.32$ used in the preceding section to yield the correct $m^{*}$ at melting pressure. However, to allow for a comparison with our results for the incompressible lattice, we solve (72) self-consistently for the cutoff parameters $r_{c}$, with $\bar{U}=1.3157$ at melting pressure, using the model form for $f_{0}^{s}(r)$ in (66). This yields $r_{c}=3.29 \AA$, which we assume to be roughly independent of pressure. The total input into the calculation is then given by the following parameters:

(i) hypothetical $v_{s}(P)$ for solid, (62).

(ii) Experimental $\kappa(P)$ for a liquid. ${ }^{25}$

(iii) $v_{m}=v\left(\boldsymbol{P}_{m}\right)$.

(iv) $m^{*} / m$ at $P_{m}$.

The pressure dependence of the effective interaction $U$ is shown in Fig. 13. It is a monotonically rising function of $P$, increasing from $\bar{U}=0.788(\hat{=} 14.4 \mathrm{~K})$ at saturated vapor pressure to $\bar{U}=1.316(\hat{=} 27.6 \mathrm{~K})$ at melting pressure $P_{m}$. For $P>P_{m}$, i.e., in the regime where, experimentally, one is already in the solid phase, we have used the experimentally measured $v_{s}(P)$ (Refs. 35-37) and a smooth extrapolation of the $v(P)$ curve for the liquid as shown in Fig. 11, indicated by the dashed line $\left(v_{l}\right)$. The interaction $U$ is seen to rise further for $P>P_{m}$.

The results for the lattice-gas model with a compressible lattice are shown in Figs. 1-4 and 7-10. The deviation from half-filling, $\delta$, again monotonically decreases for increasing pressure. At $P=0$, one finds $\delta_{0}=0.186$, which is roughly half of the corresponding value for the incompressible case. The other half of the volume change of the liquid is due to the volume change of the lattice it-

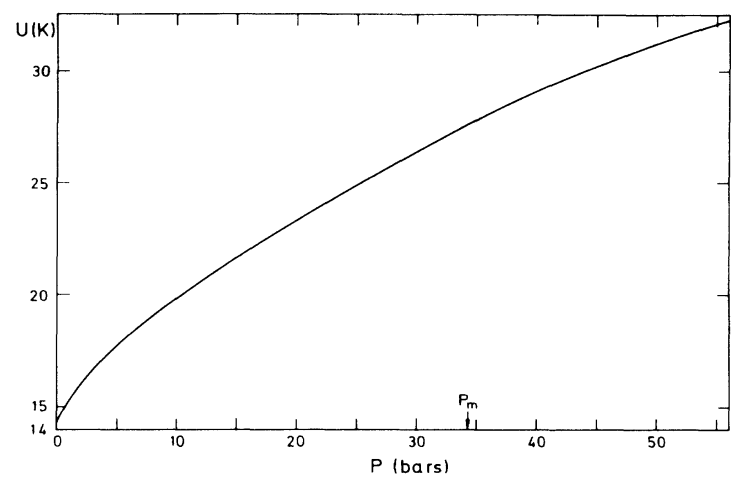

FIG. 13. The effective interaction $U$ in the lattice-gas model as calculated from the soft-core potential $f_{0}^{s}(r)$ is shown as a function of pressure.

self. For $P>P_{m}, \delta$ is seen to decrease only very slightly and, in fact, does not go to zero up to the highest pressures included in Fig. 11. This means that in the model with a compressible lattice, as considered here, $v(P)$ and $v_{s}(\boldsymbol{P})$ are approximately parallel for $\boldsymbol{P}>\boldsymbol{P}_{m}$ and do not meet (where $\delta=0$ ); even larger pressures at which the curves might meet are outside the scope of the model in any case. Similarly, $d$, the density of doubly occupied sites, decreases monotonically (Fig. 2). At $P=0, d$ is considerably larger than in the incompressible case, mainly because $\bar{U}$ is rather small. For $P>P_{m}, d$ decreases only slightly. In particular, $d$ does not vanish, i.e., there is no localization transition anymore. The relative dependence of $d$ on $\delta$ is shown in Fig. 3. Since $\delta$ and $d$ no longer vanish, a scaling property $\delta \propto d$ in the incompressible case cannot be deduced. In fact, an extrapolation of the curve would indicate that $d$ vanishes at a finite $\delta \simeq 0.035$. As discussed below (46), such a transition is only possible for infinite $U$.

The absence of a transition $d \rightarrow 0$ is clearly reflected in the results for $m^{*} / m, F_{0}^{s}$, and $\chi_{s}$. They no longer diverge at a finite pressure, implying a much less pronounced pressure dependence of these quantities than in the incompressible case. This is clearly demonstrated in Fig. 4, which shows the effective mass versus pressure. The increase of $m^{*} / m$ from its value 1.63 at $P=0$ to 5.76 at melting pressure is very smooth and closely resembles the experimental pressure dependence. For $P>P_{m}$ this slight increase continues since our model does not describe the first-order transition to the solid at $P_{m}$.

Using $m^{*}$ we may determine the Landau parameter $F_{0}^{s}$ (Fig. 7) from the compressibility. Again, the pressure dependence is described more realistically than in the incompressible case.

The residual pressure $P_{\text {res }}$ may actually be calculated in the present case. The result, shown in Fig. 8, is both qualitatively and quantitatively quite similar to the result of the incompressible case, where $P_{\text {res }}$ had been fitted using a $1 / v$ expansion.

The spin susceptibility is shown in Fig. 9. The pressure dependence is now much weaker than in the incompressi- 
ble case. On the other hand, the Landau parameter $F_{0}^{a}$ (Fig. 10) is found to be more pressure dependent, particularly at low pressures. This is due to the fact that the effective mass has a stronger pressure dependence at low pressures than in the experiment.

\section{CONCLUSION}

Using the results of the Gutzwiller approach ${ }^{14,15}$ to the Hubbard model, we have investigated a lattice-gas model with variable density. The model is intended to describe ground-state properties of normal liquid ${ }^{3} \mathrm{He}$. For this purpose we assumed the existence of a lattice on which particles interact. The filling factor $n$ is arbitrary; it is measured in terms of $\delta=1-n$, i.e., the deviation from the case of a half-filled band. While the solid is characterized by $d=\delta=0$ (localized, half-filled limit, where $d$ is the density of doubly occupied sites), the liquid state has $d, \delta>0$. Introducing the concept of a pressure yields an equation governing the filling factor in the liquid. The external pressure acts both on the interacting particles and on the lattice itself. Neglecting the effect on the lattice, i.e., assuming an incompressible lattice (and, hence, a pressure-independent on-site interaction $U$ ), the change in molar volume of the liquid is solely accounted for by the change in the filling factor, i.e., $\delta$. We find that, at a finite pressure $P_{c}>P_{m}$ (melting pressure), a transition to a localized state with $d=0$ and $\delta=0$ takes place. In particular, $d$ and $\delta$ are found to scale as the transition is approached. Hence, the localization transition $d=0$ occurs precisely at half-filling. This fact backs up earlier investi- gations which explicitly assumed $\delta=0$ in the almostlocalized limit. ${ }^{11,20}$ The pressure dependence of the effective mass, the spin susceptibility, and the compressibility are calculated. The first two quantities are found to be strongly enhanced, while the latter is strongly reduced compared to the noninteracting system. At pressures $P \lesssim P_{m}$, a pronounced pressure dependence due to the incipient localization transition in the model is obtained.

In a second model, the effect of the external pressure on the underlying lattice is taken into account by allowing for a finite compressibility of the lattice. The model repulsion $U$ is connected to the microscopic soft-core potential $^{38} f_{0}^{s}(r)$ by using the experimentally known compressibility of the liquid. This allows one to calculate the pressure dependence of the effective mass and the spin susceptibility. As before, both quantities are considerably enhanced because both $d$ and $\delta$ are small. However, an actual transition no longer occurs. This, in turn, removes the strong pressure dependence at high pressures found in the case of an incompressible lattice and yields a smooth increase with pressure, as qualitatively observed in the experiment.

\section{ACKNOWLEDGMENTS}

We gratefully acknowledge the hospitality of the Aspen Center for Physics where the present work was started. We wish to thank T. M. Rice for valuable discussions concerning the role of interstitials in a lattice model for a liquid. One of us (D.V.) acknowledges financial support from the Deutsche Forschungsgemeinschaft.
*Also at Max-Planck-Institut für Physik und Astrophysik, D8000 München 40, Federal Republic of Germany. Present address: University of Florida, Physics Department, Gainesville, FL 32611.

${ }^{1}$ L. D. Landau, Zh. Eksp. Teor. Fiz. 30, 1058 (1956) [Sov. Phys. - JETP 3, 920 (1957)].

${ }^{2}$ G. Baym and C. J. Pethick, in The Physics of Liquid and Solid Helium, Part II, edited by K. H. Bennemann and J. B. Ketterson (Wiley, New York, 1978).

${ }^{3}$ G. A. Stewart, Rev. Mod. Phys. 56, 755 (1984).

${ }^{4}$ P. A. Lee, T. M. Rice, J. W. Serene, L. J. Sham, and J. W. Wilkins, Comments Condens. Mater. Phys. 12, 99 (1986).

${ }^{5}$ C. J. Pethick and D. Pines, in Proceedings of the 4th International Conference on Recent Progress in Many-Body Theories, edited by P. Siemens and R. Smith (Springer-Verlag, Berlin, 1986), and unpublished.

${ }^{6}$ P. A. Lee and T. V. Ramakrishnan, Rev. Mod. Phys. 57, 287 (1985).

${ }^{7}$ T. M. Rice, Philos. Mag. B 52, 419 (1985).

${ }^{8}$ G. A. Thomas, Philos. Mag. B 52, 479 (1985).

${ }^{9}$ A. J. Leggett, Rev. Mod. Phys. 47, 331 (1975).

${ }^{10}$ J. C. Wheatley, Rev. Mod. Phys. 47, 415 (1975).

${ }^{11} \mathrm{P}$. W. Anderson and W. F. Brinkman, in The Physics of Liquid and Solid Helium, Part II, Ref. 2.

12P. Wölfle, Rep. Prog. Phys. 42, 269 (1979).

${ }^{13} \mathrm{P}$. Wölfle and D. Vollhardt, The Superfluid Phases of ${ }^{3} \mathrm{He}$ (Francis and Taylor, London, in press).

${ }^{14}$ M. C. Gutzwiller, Phys. Rev. Lett. 10, 159 (1963).
${ }^{15}$ M. C. Gutzwiller, Phys. Rev. 137, A1726 (1965).

16J. Hubbard, Proc. R. Soc. London, Ser. A 276, 238 (1963).

${ }^{17}$ J. Kanamori, Prog. Theor. Phys. 30, 275 (1963).

${ }^{18}$ W. F. Brinkman and T. M. Rice, Phys. Rev. B 2, 4302 (1970).

${ }^{19}$ T. M. Rice and W. F. Brinkman in Alloys, Magnets and Superconductors, edited by R. E. Mills, E. Ascher, and R. Jaffee (McGraw-Hill, New York, 1971), p. 593.

${ }^{20}$ D. Vollhardt, Rev. Mod. Phys. 56, 99 (1984).

${ }^{21}$ E. Feenberg, Theory of Quantum Fluids (Academic, New York, 1968).

${ }^{22}$ G. Kotliar and A. E. Ruckenstein, Phys. Rev. Lett. 57, 1362 (1986).

${ }^{23}$ J. M. Luttinger, Phys. Rev. 119, 1153 (1960).

${ }^{24}$ K. Levin and O. T. Valls, Phys. Rep. 98, 1 (1983).

${ }^{25}$ D. S. Greywall, Phys. Rev. B 27, 2747 (1983).

${ }^{26}$ M. Pfitzner and P. Wölfle, Phys. Rev. B 33, 2003 (1986).

${ }^{27}$ S. Babu and G. E. Brown, Ann. Phys. (N.Y.) 78, 1 (1973).

${ }^{28}$ T. L. Ainsworth, K. S. Bedell, G. E. Brown, and K. F. Quader, J. Low Temp. Phys. 50, 319 (1983).

${ }^{29}$ K. S. Bedell and K. F. Quader, Phys. Rev. B 32, 3296 (1985).

${ }^{30}$ D. Baeriswyl, C. Gros, and T. M. Rice (unpublished).

${ }^{31}$ K. Seiler, C. Gros, T. M. Rice, K. Ueda, and D. Vollhardt, J. Low Temp. Phys. 64, 195 (1986).

${ }^{32}$ L. Goldstein, Phys. Rev. 96, 1455 (1954).

${ }^{33}$ A. M. Dyugaev, Zh. Eksp. Teor. Fiz. 87, 1232 (1984) [Sov. Phys._JETP 60, 704 (1984)]; assuming a particular temperature dependence of the entropy to analyze the results by Greywall (Ref. 25), this author obtains a somewhat larger spin 
entropy per particle.

${ }^{34}$ E. R. Grilly, J. Low Temp. Phys. 4, 615 (1971).

${ }^{35}$ S. G. Sydoriak, R. L. Mills, and E. R. Grilly, Phys. Rev. Lett. 4, 495 (1960).

${ }^{36}$ D. O. Edwards, J. L. Baum, D. F. Brewer, J. G. Daunt, and A. S. McWilliams, Helium Three (Ohio State University Press, Columbus, OH, 1960).
${ }^{37}$ J. H. Hetherington, W. J. Mullin, and L. H. Nosanow, Phys. Rev. 154, 175 (1967).

${ }^{38}$ C. H. Aldrich III and D. Pines, J. Low Temp. Phys. 32, 689 (1978).

${ }^{39}$ H. G. Bennewitz, G. H. Russe, H. Böhmann, D. E. Oates, and W. Schrader, Z. Phys. 253, 435 (1972). 